\title{
How strongly are the magnetic anisotropy and coordination numbers correlated in lanthanide based molecular magnets?
}

\author{
TULIKA GUPTA and GOPALAN RAJARAMAN* \\ Department of Chemistry, Indian Institute of Technology Bombay, Powai, Mumbai 400 076, India \\ e-mail: rajaraman@chem.iitb.ac.in
}

MS received 7 May 2014; revised 11 June 2014; accepted 29 June 2014

\begin{abstract}
Ab initio CASSCF+RASSI-SO investigations on a series of lanthanide complexes $\left[\mathrm{Ln}^{\mathrm{III}}=\mathrm{Dy}(\mathbf{1})\right.$, $\mathrm{Tb}(\mathbf{2}), \mathrm{Ce}(\mathbf{3}), \mathrm{Nd}(\mathbf{4}), \operatorname{Pr}(\mathbf{5})$ and $\mathrm{Sm}(\mathbf{6})]$ have been undertaken and in selected cases (for 1, 2, 3 and 4) coordination number (C.N.) around the $\mathrm{Ln}^{\text {III }}$ ion has been gradually varied to ascertain the effect of C.N. on the magnetic anisotropy. Our calculations reveal that complex $\mathbf{3}$ possesses the highest barrier height for reorientation of magnetisation $\left(\mathrm{U}_{\text {eff }}\right)$ and predict that $\mathbf{3}$ is likely to exhibit Single Molecule Magnet (SMM) behaviour. Complex $\mathbf{5}$ on the other hand is predicted to preclude any SMM behaviour as there is no intrinsic barrier for reorientation of magnetization. Ground state anisotropy of all the complexes show mixed behaviour ranging from pure Ising type to fully rhombic behaviour. Coordination number around the lanthanide ion is found to alter the magnetic behaviour of all the lanthanide complexes studied and this is contrary to the general belief that the lanthanide ions are inert and exert small ligand field interaction. High symmetric low-coordinate $\mathrm{Ln}^{\mathrm{III}}$ complexes are found to yield large $\mathrm{U}_{\text {eff }}$ values and thus should be the natural targets for achieving very large blocking temperatures.
\end{abstract}

Keywords. Lanthanides; magnetic anisotropy; coordination number; effective energy barrier, single molecule magnet.

\section{Introduction}

Since past two decades, Single Molecule Magnets (SMMs) have received appreciable attention owing to their potential applications for the use of high-density magnetic memories, magnetic refrigeration, molecular spintronics and quantum computing devices. ${ }^{1-6}$ These potential applications are due to their slow relaxation of magnetization and enhanced effective barrier heights $\left(\mathrm{U}_{\text {eff }}\right)$ for reorientation of magnetization (to date the highest $\mathrm{U}_{\text {eff }}$ reported is $\left.652 \mathrm{~cm}^{-1}\right){ }^{7}$ These behaviours can be observed when the molecule has large ground spin state with a uniaxial magnetic anisotropy, namely negative zero-field splitting (ZFS) parameter. ${ }^{6,7}$ Besides a large ground state, to increase the SMMs energy barrier and blocking temperatures, it is important to perceive a way to control the magnetic anisotropy. ${ }^{8}$ Of particular interest in recent years in research area of $\mathrm{SMMs}^{9}$ is exploration of systems containing only one spin carrier within a molecule and if such molecules exhibit magnetization blockade, these are called as Single Ion Magnets (SIMs). ${ }^{10-13}$ Large unquenched orbital angular momentum, significant intrinsic spin-orbit coupling and presence of large number of unpaired electrons make the lanthanides suitable candidates for

*For correspondence attaining large spin-reversal barriers. Besides this, area lanthanide(III) complexes also have important applications in diagnostic and therapeutic radiopharmaceuticals $^{14}$ and as luminescent probe to investigate biomedical systems. ${ }^{15}$

In SIMs, magnetic properties are directly connected with the crystal field created by the surrounding ligands. This emphasizes the importance of crystal field environment in the design of novel SIMs. ${ }^{16-19}$ In SIMs coordination environment, number, local point group symmetry and ligand field strength together need to be manipulated to obtain large energy barrier. Magnetic anisotropy in these SIMs arise due to interaction between single metal ion and the ligand field, resulting in preferential orientation of the magnetic moment. ${ }^{20}$ This eventually generates a strong influence of coordination geometries on SIM properties. Thus, magnetic properties are affected not only by structural and electronic features of the molecule, but also by their surroundings resulting in the spin dynamics method in SIMs to be complex and poorly understood problem. ${ }^{21}$ Indeed, these processes can have significant influence on the field dependence of the relaxation time ${ }^{22}$ as well as field-induced multiple relaxation processes. ${ }^{24}$ Moreover, Lanthanide containing SIMs are attractive for testing synthetic design principles for slow paramagnetic relaxation because their properties can be tuned at will. In general, in a given 
crystal field, the ground magnetic state of the complex (characterized by the total angular momentum $J$ ), splits into $\pm \mathrm{M}_{J}$ sub-levels. In some instances, this leads to a $\mathrm{J}$-splitting in which the levels with higher $\mathrm{M}_{J}$ values are stabilized with respect to the levels with lower $\mathrm{M}_{J}$ values. This leads to the appearance of a barrier between the $\mathrm{M}_{J}$ levels explaining superparamagnetic blocking in the SIMs.

Despite enormous synthetic effort expended in making novel examples of lanthanide-based SIMs, clear understanding of the origin of the slow relaxation of the magnetisation and the mechanisms of the Quantum Tunnelling of the Magnetisation (QTM) still remains scarce. Understanding the factors determining the formation of efficient barriers of blocking of magnetization in SIMs is of primary importance for the fast advance of this new research area. ${ }^{9,25,26}$ Slow relaxation of magnetization in SIMs can be achieved by effective involvement of anisotropy at the metal sites. Extensive studies ${ }^{27}$ on these SIMs based on lanthanides prove their importance due to their large spin-orbit coupling compared to the crystal-field splitting of the magnetic $4 \mathrm{f}$-shell. ${ }^{28}$ All these studies have concluded the crucial participation of excited states of the lanthanide ions in the relaxation process of both mononuclear and polynuclear complexes. ${ }^{16,19,29-34}$

The anisotropy axis on the metal ions can be easily determined if the metal centres possess some symmetry elements. Using the angular overlap model ${ }^{35}$ zerofield splitting and the direction of anisotropy axis can be estimated. However such qualitative ligand-field theories are extremely complex ${ }^{36}$ for lanthanides complexes rendering less accurate predictions. Although numerous experimental tools such as Inelastic Neutron scattering (INS), ${ }^{37,38}$ multifrequency high-field EPR, ${ }^{39,40}$ field and orientation dependent magnetic susceptibility have been used to investigate the magnetic anisotropy, none of them suffice to resolve the directions of local anisotropy axes accurately.

The only straightforward way to attain quantitative information about magnetic networks is via fragment quantum chemistry calculations taking spin-orbit coupling into account non-perturbatively. Particularly, the direction of the local anisotropy axis on a metal site is easily gained as the main magnetic axis of the $g$ tensors of the corresponding Kramers doublet. The single-ion properties of the $4 \mathrm{f}$ metal ions are difficult to depict due to the shielded nature of the $4 \mathrm{f}$ orbitals, resulting in weak exchange interactions. Recent advances in post Hartree-Fock multi-configurational $a b$ initio methodology have made accurate quantum chemical calculations on paramagnetic $4 \mathrm{f}$ compounds possible. ${ }^{41}$ The Complete Active Space Self Consistent Field (CASSCF) method can accurately predict the magnetic properties of lanthanide complexes, ${ }^{42,43}$ and calculations of this type have become an indispensable tool for the explanation of increasingly interesting magnetic phenomena. ${ }^{44}$ Although CASSCF $a b$ initio calculations are extremely versatile and implicitly include all the effects required to elucidate the magnetic properties, the results offer little in the way of chemically intuitive explanations and attainment of reliable results often requires considerable intervention by expert theorists equipped with access to powerful computational resources.

Although higher coordination number around the lanthanide ions are preferred, low coordination number lanthanide complexes had been reported as early as in 1990 's, ${ }^{65}$ despite no such detailed magnetic study on these complexes. Recently, Tang and co-workers have reported low-valent complexes showing slow magnetisation of relaxation. ${ }^{66}$ In the field of lanthanide based SIMs, recently researchers have focussed on two directions: how to enhance the effective barrier height for reorientation of magnetization and to explain the mechanism of versatile thermal relaxation taking place during the reorientation. As crystal field parameter, coordination number and ligation around the $\mathrm{Ln}^{\mathrm{III}}$ ion play crucial role in determining the direction of anisotropy, we have decided to play with these parameters with the hope of understanding the aforementioned key points employing computational tools. Considering the recent research trend on lanthanide based SIMs we intend to offer explicit understanding of anisotropy and magnetic relaxation in varied coordination and metallic environment. Hence, here we have performed detailed $a b$ initio calculations on monometallic $\mathrm{Ln}^{\mathrm{III}}\left[\mathrm{Ln}^{\mathrm{III}}=\right.$ $\operatorname{Dy}(\mathbf{1}), \operatorname{Tb}(\mathbf{2}), \mathrm{Ce}(\mathbf{3}), \operatorname{Nd}(\mathbf{4}), \operatorname{Pr}(\mathbf{5})$ and $\operatorname{Sm}(\mathbf{6})]$ crystal structures surrounded by nitrate ligands. Among these, ions with odd number of electrons and half integral angular momentum values $(\mathrm{J})$ are known as Kramers ions (Dy ${ }^{\mathrm{III}}, \mathrm{Ce}^{\mathrm{III}}, \mathrm{Nd}^{\mathrm{III}}$ and $\mathrm{Sm}^{\mathrm{III}}$ ) whereas ions with even number of electrons and integral angular momentum values $(\mathbf{J})$ are known as non-Kramers ions $\left(\mathrm{Tb}^{\mathrm{III}}\right.$ and $\left.\mathrm{Pr}^{\mathrm{III}}\right)$. We have calculated effective barrier height, direction of anisotropy for this series. In selected cases, (for 1, 2, 3 and 4) we have gradually varied the coordination number (C.N.) around $\mathrm{Ln}^{\text {III }}$ ions from C.N. 12 to C.N. 2 with an aim to observe the effect of coordination number on the magnetic properties of these lanthanide complexes. To keep the same coordination environment across all models structures, all the complexes are modelled with nitrate ions, however synthesis of such complexes would require monodentate ligands such as hydroxide or other ligands. 


\section{Computational Methodology}

MOLCAS $7.8^{68}$ program package has been used to perform post-Hartree-Fock $a b$ initio calculations. Using multiconfigurational approach, relativistic effects were treated in two steps, based on Douglas-Kroll Hamiltonian. For the generation of basis sets, scalar terms were included which has been used to determine spin-free wave functions and also energies through the use of the complete active space self-consistent field (CASSCF) method. So, spin-orbit free states were obtained by employing the RASSCF method whereas spin-orbit coupling has been taken into account using RASSI-SO method $^{69}$ which uses CASSCF wave functions as the basis sets and multiconfigurational wave functions as input states. The resultant wave functions and energies of the molecular multiplets were used for the calculation of the anisotropic magnetic properties and g tensors of the lowest state using a specially designed routine SINGLE-ANISO.$^{70}$ As a consequence, the magnetic properties of a single magnetic ion are calculated by $a b$ initio approach in which the spin-orbit coupling is considered non-perturbatively. We have employed [ANORCC...7s6p4d2f.] basis set for $\operatorname{Ln}^{\mathrm{III}}\left\{\mathrm{Ln}^{\mathrm{III}}=\mathrm{Dy}, \mathrm{Tb}\right.$, $\mathrm{Ce}, \mathrm{Nd}, \mathrm{Pr}$ and $\mathrm{Sm}$, [ANO-RCC... 3s2p.] basis set for $\mathrm{N}$ and [ANO-RCC... 2s.] basis set for $\mathrm{H}$ throughout our calculations. These ANO-RCC-VDZ basis sets used for all the atoms are taken from ANO-RCC basis library included in MOLCAS 7.8 program package. The active space for $\left[\mathrm{Dy}{ }^{\mathrm{III}}\left(\mathrm{NO}_{3}\right)_{6}\right]^{3-}(\mathbf{1})$, is 9 in 7 for the $\mathrm{f}^{9}$ configuration of Dy ${ }^{I I I}$ ion. Here we have employed the CASSCF calculations coupled with RASSI-SO procedure to estimate the ground state anisotropy and other parameters which controls the magnetic properties of this complex. In the configurational Interaction (CI) procedure, 21sextets, 140 quartets and 150 doublets are considered. In the RASSI module, 21 sextets, 128 quartets and 130 doublets are mixed by spin-orbit coupling. The active space for $\left[\mathrm{Tb}^{\mathrm{III}}\left(\mathrm{NO}_{3}\right)_{6}\right]^{3-}[2]$ is 8 in 7 for the $\mathrm{f}^{8}$ configuration of the $\mathrm{Tb}^{\mathrm{III}}$ ion. In the CI procedure, the septets are given 7 roots, the quintets are given 140 roots and the triplets are given 195 roots. The singlet states were not included due to computational limitations. In the RASSI module all the states within the energy window of about $40,000 \mathrm{~cm}^{-1}$ (seven septets, 105 quintets and 112 triplets) were allowed to mix by spin-orbit coupling. Cholesky decomposition of the two-electron integrals was performed to save disk space. The active space for $\left[\mathrm{Ce}^{\mathrm{III}}\left(\mathrm{NO}_{3}\right)_{6}\right]^{3-}[3]$ is 1 in 7 for the $\mathrm{f}^{1}$ configuration of $\mathrm{Ce}^{\mathrm{III}}$ ion. The CI procedure includes 7 doublet and all of them are incorporated in the RASSI module. Our next complex of interest is $\left[\mathrm{Nd}^{\mathrm{III}}\left(\mathrm{NO}_{3}\right)_{6}\right]^{3-}[4]$ which has active space of 3 in 7 for $\mathrm{f}^{3}$ electronic configuration of $\mathrm{Nd}^{\mathrm{III}}$ ion. In the CI procedure 35 quartets and 112 doublets are considered. In the RASSI module all the 35 quartets and 112 doublets are mixed by spin-orbit coupling. Our next complex of interest is $\left[\mathrm{Pr}^{\mathrm{III}}\left(\mathrm{NO}_{3}\right)_{6}\right]^{3-}[5]$ which has active space of 2 in 7 for $\mathrm{f}^{2}$ electronic configuration of $\mathrm{Pr}^{\mathrm{III}}$. In the CI procedure, triplets are given 21 roots and singlets are given 28 roots. In the RASSI module all the 21 triplets and 28 singlets are mixed by spin-orbit coupling. Now, we will focus on complex $\left[\mathrm{Sm}^{\mathrm{III}}\left(\mathrm{NO}_{3}\right)_{6}\right]^{3-}[\mathbf{6}]$ which has active space of 5 in 7 for $\mathrm{f}^{5}$ electronic configuration of $\mathrm{Sm}^{\text {III }}$ ion. The RASSI and CI calculation for $\mathrm{Sm}^{\mathrm{III}}$ is similar to that for Dy ${ }^{\mathrm{III}}$. Structural optimization have been computed using DFT calculations employing Gaussian $09^{45}$ suite. Here we have employed the B3LYP ${ }^{46-48}$ functional, along with the Cundari-Stevens double $\zeta$ polarization basis set ${ }^{49}$ for the $\mathrm{Ln}^{\mathrm{III}}$ ions and the Ahlrichs triple $\zeta$ basis set ${ }^{50}$ for the rest of the atoms.

\section{Result and Discussion}

Here we have chosen six structures 1-6 for our study. All the complexes comprise twelve coordinate Dy ${ }^{\text {III }}$, ${ }^{51}$ $\mathrm{Tb}^{\mathrm{III}},{ }^{52} \mathrm{Ce}^{\mathrm{III}},{ }^{53} \mathrm{Nd}^{\mathrm{III}},{ }^{54} \mathrm{Pr}^{\mathrm{III} 55}$ and $\mathrm{Sm}^{\mathrm{III}}$ in slightly distorted octahedron coordination environment surrounded by six symmetrically equivalent $\mathrm{NO}_{3}^{-}$ligands (figure 1). The reported X-ray structure is taken as-is for calculations for all other structures except complex 6. For complex 6, the $\mathrm{Ce}^{\mathrm{III}}$ analogue has been employed to model the $\mathrm{Sm}^{\mathrm{III}}$ structure.

Among the lanthanide family, Dy ${ }^{\mathrm{III}}$ ion has indisputably led to the largest number of pure SIMs. ${ }^{10,56-58}$ The explanation resides in the reduced QTM observed in these systems compared to other lanthanide ions owing to its large magnetic moment and odd electron configuration. Here we start our discussion with individual lanthanide ions, and compare between lanthanide complexes later.

\subsection{Studies on complex 1}

The ground state Kramers Doublet (KD) is Ising in nature $\left(g_{x x}=0.04, g_{y y}=0.06, g_{z z}=15.34\right)$ for $\mathbf{1}$. The first excited state KD possesses a large amount of transverse anisotropy and deviates largely with respect to the ground state anisotropy direction.

The energy spectrum and g-tensors for the first two Kramers doublets of the ground ${ }^{6} \mathrm{H}_{15 / 2}$ multiplet of the Dy ${ }^{\text {III }}$ ion in compound $\mathbf{1}$, are shown in table 1 , with excited multiplet ${ }^{6} \mathrm{H}_{13 / 2}$ lying at $3650 \mathrm{~cm}^{-1}$. Effective energy barrier for relaxation of magnetization $\left(\mathrm{U}_{\text {eff }}\right.$ or $\Delta \mathrm{E}$ ) in $\mathbf{1}$ is found to be $27.93 \mathrm{~cm}^{-1}$ (table 1) and this is 

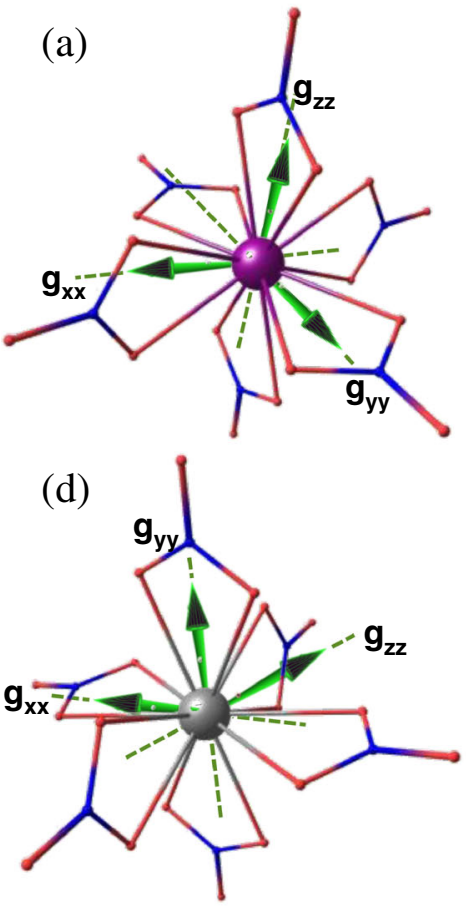

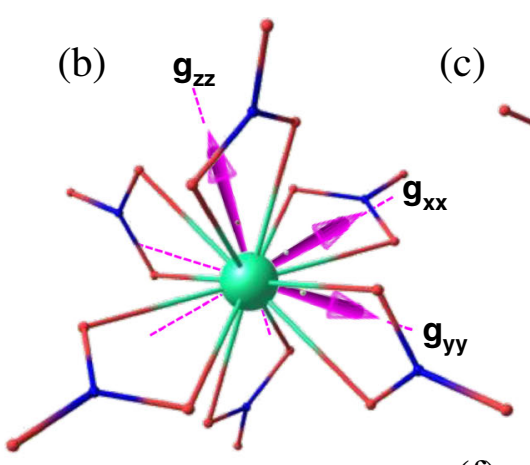

(c)

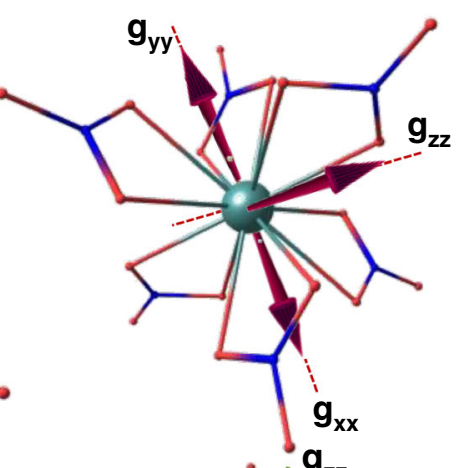

(e)

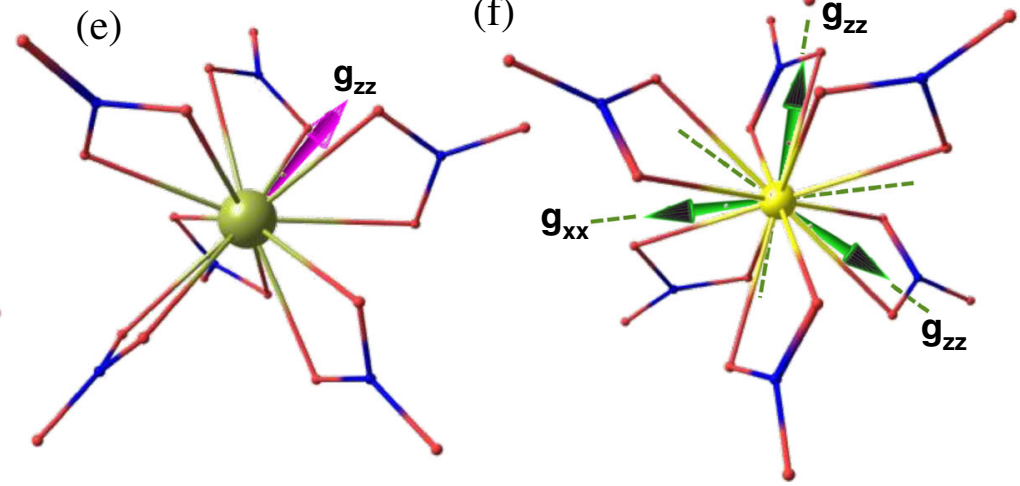

Figure 1. Showing direction of ground state anisotropy axis along $\mathrm{X}, \mathrm{Y}$ and $\mathrm{Z}$ directions in [ $\mathrm{Ln} \mathrm{n}^{\mathrm{III}}$ $\left.\left(\mathrm{NO}_{3}\right)_{6}\right]^{3-}\left\{\right.$ where $\left.\operatorname{Ln}^{\mathrm{III}}=\mathrm{a}\right) \operatorname{Dy}(\mathbf{1})$, b) $\mathrm{Tb}(\mathbf{2})$, c) $\mathrm{Ce}(\mathbf{3})$, d) $\mathrm{Nd}(\mathbf{4})$ and f) $\left.\operatorname{Sm}(\mathbf{6})\right\}$. Exceptionally, for complex 5 anisotropy only along the $\mathrm{Z}$ direction $\left(\mathrm{g}_{\mathrm{zz}}\right)$ is shown for the fourth excited state, see text for details. (atomic colour scheme: Dy ${ }^{\mathrm{III}}$-purple, $\mathrm{Tb}^{\mathrm{III}}$-green, $\mathrm{Ce}^{\mathrm{III}}$-aqua, $\mathrm{Nd}^{\mathrm{III}}$-silver, $\mathrm{Pr}^{\mathrm{III}}$-yellowish green, $\mathrm{Sm}^{\mathrm{III}}$-yellow, O-red, N-blue).

in line with the experimental value reported for a similar structure.

\subsection{Variation of coordination number (C. N.) on Dy ${ }^{I I I}$}

Pronounced dependence of magnetic anisotropy on the nature of counter-ions, ligands around the lanthanide ion, etc have been reported. ${ }^{59-61}$ These results suggest that the magnetic behaviour of lanthanides depend on the structural changes around the ion. ${ }^{59-61}$ Considering all these points, we have decided to extend our study by varying the coordination number gradually from 12 to 2 around the lanthanide ion to observe the effect of ligation on the magnetic properties in complex 1-4. All the structures possessing C. N. 11 to 2 are optimised using Gaussian $09^{45}$ software suite (see section 2).

Analysing the changes of magnetic properties around Dy ${ }^{\mathrm{III}}$ upon variation of C. N. (table 2 and figure $3 \mathrm{~b}$ ) we found that, among all the C. N. models tested, model with C. N. 11 shows the strongest transverse anisotropy. As we decrease the C. N. around Dy ${ }^{\mathrm{III}}$, a

Table 1. Calculated energy spectrum, g tensors, relative energies and angles $(\theta)$ of the principal anisotropy axes of first excited states with respect to the ground state for ground and fist excited Kramers (for 1,3,4,6) and pseudo doublets (for 2 and 5) in 1-6.

\begin{tabular}{|c|c|c|c|c|c|c|}
\hline Ground KDs /Non-KDs & 1 & 2 & 3 & 4 & 5 & 6 \\
\hline$g_{x x}$ & 0.04 & 0 & 0.08 & 0.58 & - & 0.11 \\
\hline $\mathrm{g}_{\mathrm{yy}}$ & 0.06 & 0 & 0.53 & 1.73 & - & 0.47 \\
\hline $\mathrm{g}_{\mathrm{zz}}$ & 15.34 & 14.42 & 3.70 & 3.66 & - & 1.06 \\
\hline Relative energy $\left(\mathrm{cm}^{-1}\right)$ & 0.0 & 0.0 and 4.33 & 0.0 & 0.0 & 0.0 & 0.0 \\
\hline $1^{\text {st }}$ excited KDs $/$ Non-KDs & 1 & 2 & 3 & 4 & 5 & 6 \\
\hline$g_{x x}$ & 8.60 & 0 & 0.01 & 0.51 & 0 & 0.88 \\
\hline $\mathrm{g}_{\mathrm{yy}}$ & 8.56 & 0 & 0.94 & 0.70 & 0 & 0.59 \\
\hline $\mathrm{g}_{\mathrm{zz}}$ & 5.35 & 10.18 & 2.55 & 3.77 & 0 & 0.27 \\
\hline Relative energy $\left(\mathrm{cm}^{-1}\right)$ & 27.93 & 15.17 and 18.66 & 107.90 & 16.20 & 25.83 & 90.03 \\
\hline Angle $\{\theta\}\left(^{\circ}\right)$ & 0.10 & 98.60 & 40.41 & 85.30 & - & 52.78 \\
\hline
\end{tabular}


Table 2. Calculated energy spectrum, g tensors and angles $(\theta)$ of the principal anisotropy axes of ground KDs of low valent Dy ${ }^{\mathrm{III}}$ model complexes (from 11 to 2 C.N. change around Dy ${ }^{\mathrm{III}}$ ion) with respect to their respective first excited state KDs.

\begin{tabular}{lrllcr}
\hline $\begin{array}{l}\text { Coordination no. } \\
\text { around Dy in 1 }\end{array}$ & $\mathrm{U}_{\text {eff }}\left(\mathrm{cm}^{-1}\right)$ & $\mathrm{g}_{\mathrm{x}}$ & $\mathrm{g}_{\mathrm{y}}$ & $\mathrm{g}_{\mathrm{z}}$ & Angle $\{\theta\}\left(^{\circ}\right)$ \\
\hline 12 & 27.93 & 0.04 & 0.06 & 15.34 & 0.10 \\
11 & 49.02 & 1.88 & 5.04 & 14.18 & 63.90 \\
10 & 19.67 & 0.24 & 2.06 & 16.17 & 49.55 \\
9 & 41.43 & 0.06 & 0.27 & 17.32 & 103.75 \\
8 & 120.17 & 0.02 & 0.05 & 19.63 & 80.08 \\
7 & 28.76 & 2.78 & 3.89 & 12.38 & 33.21 \\
6 & 11.68 & 1.0 & 1.60 & 17.82 & 72.79 \\
5 & 52.56 & 0.72 & 2.83 & 17.24 & 61.45 \\
$4^{*}$ & 420.04 & 0.0 & 0.0 & 19.81 & 163.40 \\
$3^{*}$ & 471.15 & 0 & 0 & 19.84 & 168.25 \\
$2^{*}$ & 410.48 & 0 & 0 & 19.86 & 2.87 \\
\hline
\end{tabular}

*indicates barrier computed with second excited KDs due to collinearity with the first excited KDs.

gradual increase of Ising nature of anisotropy is attained till C. N. 8 model (figure 2). Further reduction of C. N. from 8 to 7 leads to an increase in rhombic anisotropy and for C. N. 6 and 5, a reduced rhombicity is observed (figure 3). As we go further down from C. N. 5 to lower Coordination Numbers, axial nature of the anisotropy is observed (for C. N. 2, 3 and 4 models). The angle between the direction of the excited state $\mathrm{g}_{\mathrm{zz}}$ and the ground state $\mathrm{g}_{\mathrm{zz}}$ is represented by tilt angle $\theta$. Larger $\theta$ values suggest that the relaxation is predominantly happening via that particular KDs while smaller values reveal that the anisotropy is collinear and relaxation of magnetisation is likely to proceed via higher excited states leading to larger $\mathrm{U}_{\text {eff }}$ values. ${ }^{62}$ This $\theta$ angle is found to be large for all the tested models except for models with C. N 2. Among all the C. N. models tested, model with C. N. 3 is found to possess highest $\mathrm{U}_{\text {eff }}$ values and this finding is in accordance with the recent synthesis of three coordinated Dy ${ }^{\mathrm{III}}$ complexes possessing large $U_{\text {eff }}$ value. ${ }^{63}$ This observation highlights the importance of $\mathrm{C}$. N. in fine tuning the $\mathrm{U}_{\text {eff }}$ values in lanthanide molecular magnets (figure $4 \mathrm{a}$ ). Barring a few exceptions, we can conclude that C. N. variation is inversely proportional to the magnitude of $\mathrm{U}_{\text {eff }}$ values. We have also performed analysis on formation energies of all these varied coordinated complexes (figure 3a). We found that formation of model with C. N. 11 is most unfavourable, possessing highest positive formation energy (endothermic) with respect to C. N. 12 while the formation of C. N. 10 complex is most preferred (thermodynamically most stable compared to C. N. 12). This is in accordance with the experimental report where 10 co-ordination ${ }^{53}$ Dy ${ }^{\text {III }}$ complexes are much more common than other coordination numbers. On the same note, except model with C. N. 8, formations of rest of the complexes are endothermic in nature. This is only a qualitative analysis on the stability of these models with respect to C. N. 12 as this analysis ignores the kinetics of formation and the likely variation in the ligand structures.

\subsection{Studies on complex 2}

Ground and first excited pseudo-doublet in $\mathbf{2}$ are of strong Ising nature with $\mathrm{U}_{\text {eff }}$ value computed to be 15.17 $\mathrm{cm}^{-1}$ (figure 4). Computed anisotropy for the first two pseudo doublet of the ${ }^{7} \mathrm{~F}_{6}$ multiplet of the $\mathrm{Tb}^{\mathrm{III}}$ ion in compound $\mathbf{2}$ is shown in table 1 with subsequent excited multiplet ${ }^{7} \mathrm{~F}_{5}$ lying at $1990 \mathrm{~cm}^{-1}$. The first excited pseudo-doublet forms a larger angle with respect to the ground state principal anisotropy directions. The tunnelling gap within the ground and first excited pseudo doublets are 4.33 and $3.49 \mathrm{~cm}^{-1}$ respectively.

\subsection{Variation of coordination number (C. N.) in $T b^{I I I}$}

Being inspired by the effect of C.N. on the magnetic properties of the Dy ${ }^{I I I}$ models, we have performed a thorough analysis of gradual C.N. change around $\mathrm{Tb}^{\mathrm{III}}$ in 2. Independent of the C.N. all the ground state levels in $\mathbf{2}$ is found to be strongly Ising in nature, contrary to the behaviour observed for Dy ${ }^{\mathrm{III}}$. This unfolds an interesting observation that the $\mathrm{Tb}^{\mathrm{III}}$ ground state anisotropy is unlikely to be influenced by the number of ligand atoms coordinated to the metal ions (table 3).

Among all the C. N. examined (figure 5), complex with C. N. 3 has been found to possess the largest $U_{\text {eff }}$ 


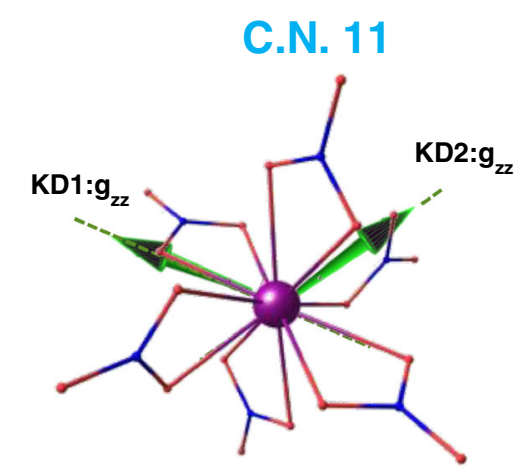

C.N. 8
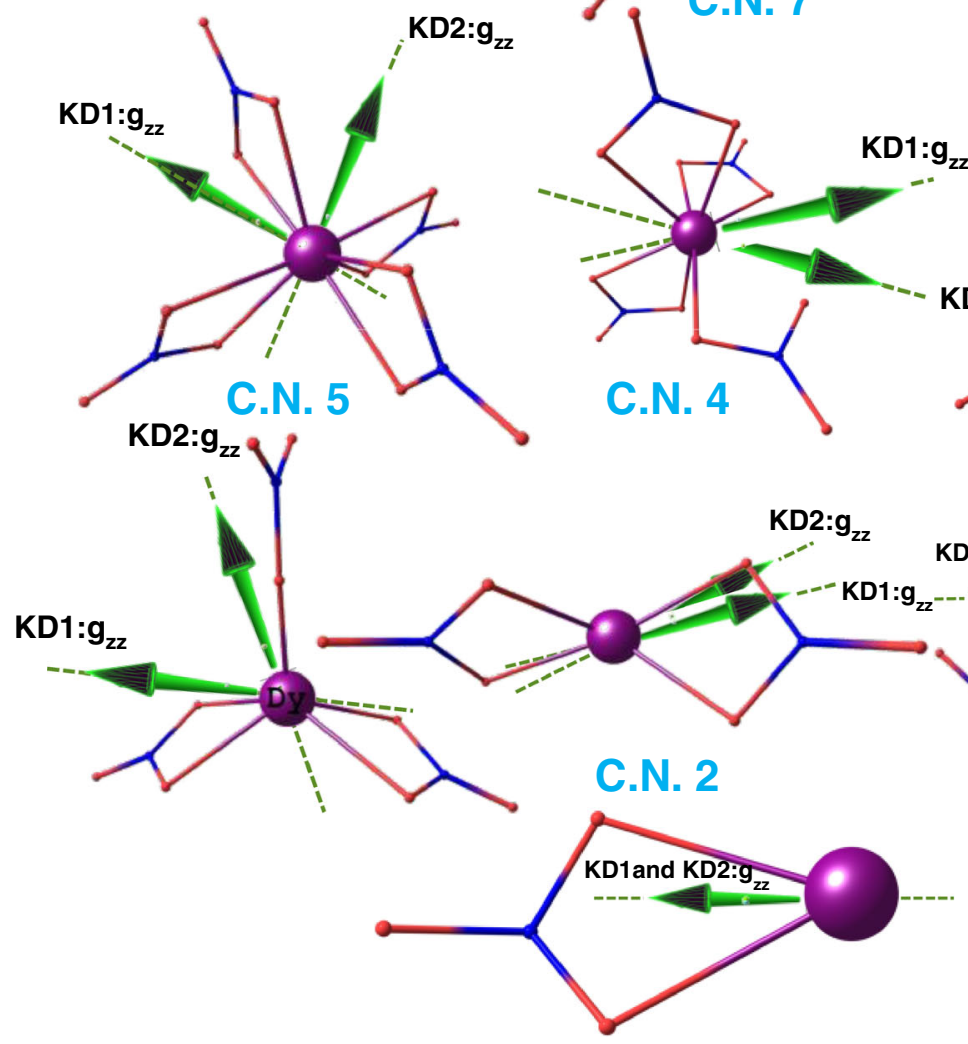

C.N. 10

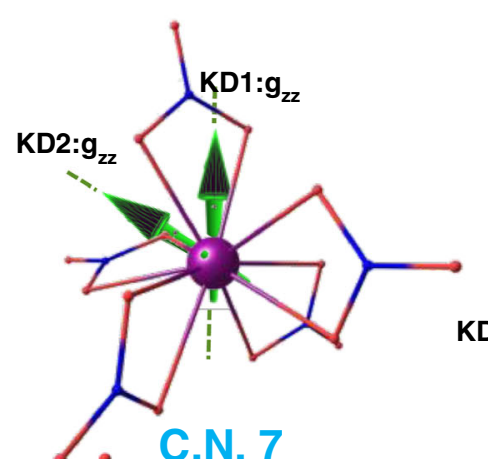

C.N. 9

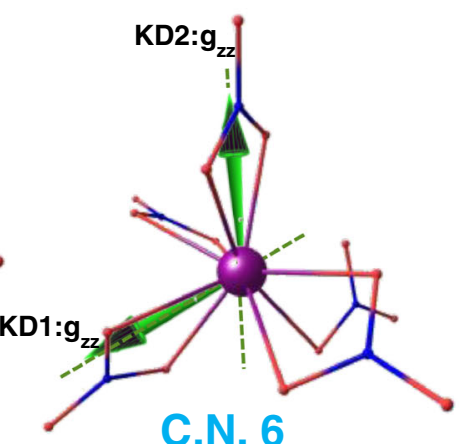

C.N. 6

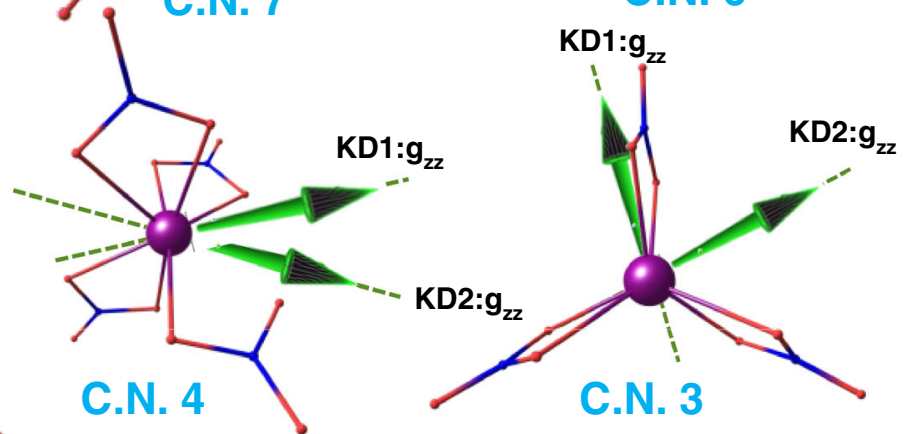

Figure 2. Model structures where C. N. varied from 11 to 2 around Dy ${ }^{I I I}$ in $\mathbf{1}$ along with the computed $\mathrm{g}_{\mathrm{zz}}$ direction for ground and first excited KD states.

values $\left(270.52 \mathrm{~cm}^{-1}\right)$. This can be supported by recent trend and interest towards the synthesis of low valent lanthanide complexes. ${ }^{63}$ The angle $\theta$ is found to be large for all the tested models. Here again, except a few cases, C. N. variation is inversely proportional to the magnitude of $U_{\text {eff }}$. Similar to complex 1, we found that the formation of complex with C. N. 11 is most unfavourable possessing highest positive formation energy (endothermic) and formation of C. N. 10 complex is most preferred. On the same note, except complexes with $\mathrm{C}$. N. 8 and 6 , formations of rest of the complexes are endothermic in nature. From figure $4 \mathrm{~b}$, we can interpret that independent of C. N., in both $\mathbf{1}$ and $\mathbf{2}$ the magnitude of the $g_{z z}$ is inversely proportional to the
$\mathrm{U}_{\text {eff }}$ value indicating intimate relationship between axial anisotropy and the relaxation energy barrier.

\subsection{Studies on Complex 3}

The energy spectrum and g-tensors for the Kramers doublets of the ground ${ }^{2} \mathrm{~F}_{5 / 2}$ multiplet of $\mathrm{Ce}^{\mathrm{III}}$ ion in $\mathbf{3}$ is shown in table 1 with excited multiplet ${ }^{2} \mathrm{~F}_{7 / 2}$ lying at $2157 \mathrm{~cm}^{-1}$ higher in energy. Both the ground and first excited KD in $\mathbf{3}$ shows strongly rhombic nature of the anisotropy with large deviation of the first excited $\mathrm{KD}$ with respect to the ground state KD. Among the series of lanthanides studied by us, complex $\mathbf{3}$ is found to possess the highest $U_{\text {eff }}$ value $\left(107.90 \mathrm{~cm}^{-1}\right)$. 


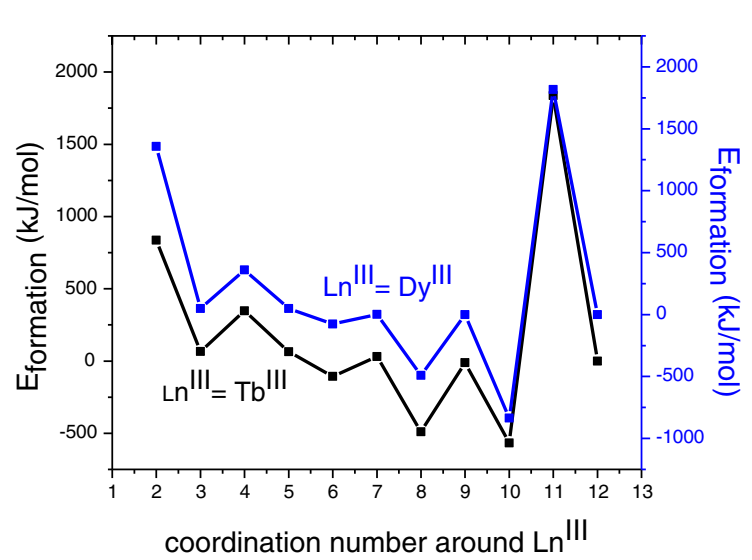

(a)

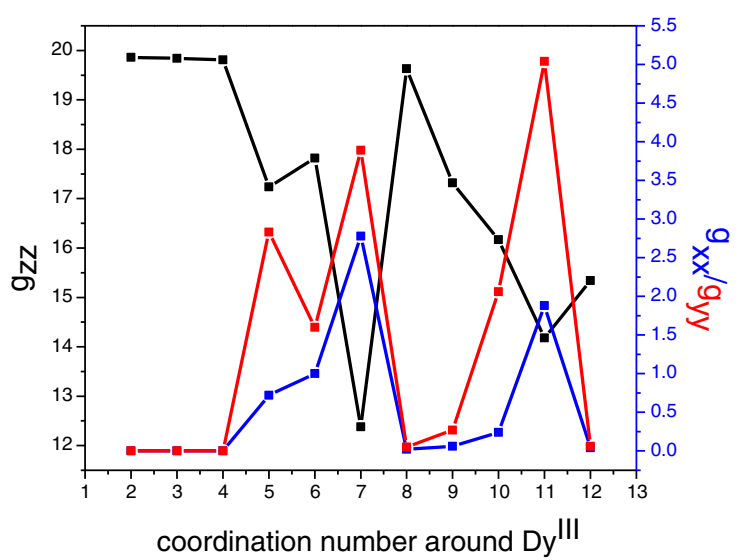

(b)

Figure 3. (a) Effect of gradual C.N. change from 12 to 2 on the formation energies for complexes 1 and 2; (b) variation in the magnitude of $g_{x x}, g_{y y}$ and $g_{z z}$ values upon gradual change of C. N. from 12 to 2 around Dy ${ }^{\text {III }}$ ions in $\mathbf{1}$.

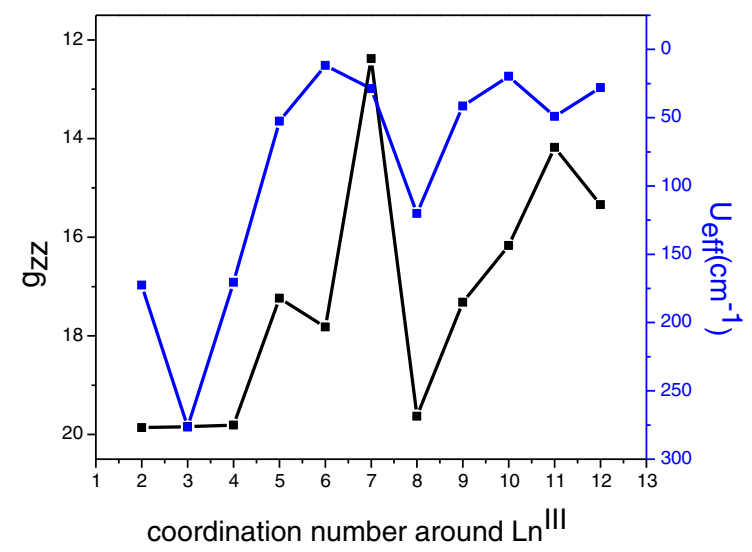

(a)

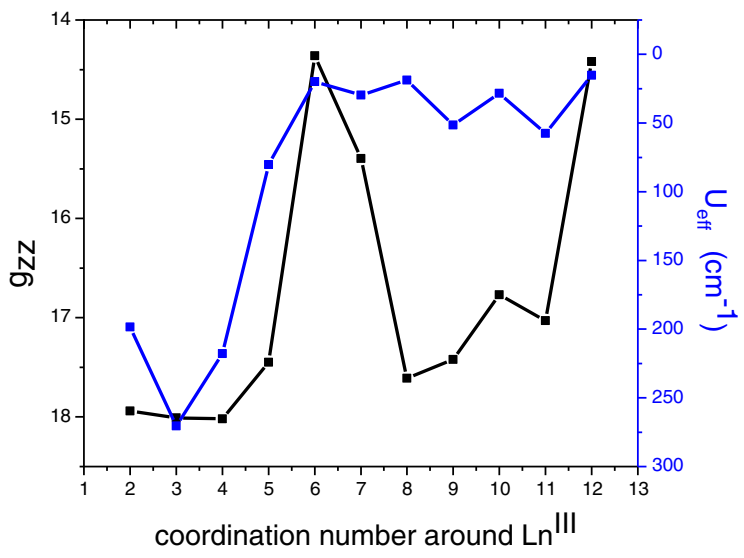

(b)

Figure 4. Dependence of C.N. on the magnitude of the effective energy barrier for relaxation along with ground state anisotropy direction along $\mathrm{Z}$ axis for (a) $\mathrm{Dy}^{\mathrm{III}}$ and; (b) $\mathrm{Tb}^{\mathrm{III}}$ ions.

\subsection{Variation of coordination number (C.N.) in $C e^{I I I}$}

All the computed g-tensors and energy spectrum for varied coordinated complexes are shown in table 4.

Among all the C. N. studied (figure 5), complex with C.N. 2 model is found to possess the largest $U_{\text {eff }}$ value. Model with C. N. 11 shows rhombic anisotropy while gradual increase in transverse nature of anisotropy is achieved till C. N. 9. Model with C.N. 7 possesses axial anisotropy followed by rhombicity in case of C.N. 6 . As we further go down from C. N. 6 to 5, transverse anisotropic behaviour is observed again. Further reduction in C.N. around $\mathrm{Ce}^{\mathrm{III}}$ in $\mathbf{3}$, produces almost Ising behaviour. Independent of the C. N., the $\theta$ angle is found to be large for all tested models. Model with C.N. 5 is found to have largest transverse anisotropy whereas C. N. 2 has largest Ising anisotropy.

\subsection{Studies on complex 4}

The energy spectrum and g-tensors for the Kramers doublets of the ground ${ }^{4} \mathrm{I}_{9 / 2}$ multiplet of $\mathrm{Nd}^{\mathrm{III}}$ ion computed for 4 is shown in table 1 with excited multiplet ${ }^{4} \mathrm{I}_{11 / 2}$ lying at $2157 \mathrm{~cm}^{-1}$ higher in energy. Ground state shows rhombic character whereas first excited state possesses axial anisotropy with $U_{\text {eff }}$ value of $16.2 \mathrm{~cm}^{-1}$. First excited anisotropy direction lies almost orthogonal with respect to the ground state Kramers Doublet.

\subsection{Variation of coordination number $\left(C . N\right.$.) in $N d^{I I I}$}

The energy spectrum and all computed values for versatile coordinated complexes are listed in table 5 .

Model with C.N. 4 is found to possess the largest $\mathrm{U}_{\text {eff }}$ and also possesses axial ground state anisotropy and 
Table 3. Calculated energy spectrum, g tensors and angles $(\theta)$ of the principal anisotropy axes of ground pseudo-doublets of $\mathrm{Tb}^{\mathrm{III}}$ model complexes (from 11 to 2 C.N. change around $\mathrm{Tb}^{\mathrm{III}}$ ion) with respect to their respective first excited state pseudo-doublets.

\begin{tabular}{lccccc}
\hline $\begin{array}{l}\text { Coordination no. } \\
\text { around } \mathrm{Tb}\end{array}$ & $\mathrm{U}_{\text {eff }}\left(\mathrm{cm}^{-1}\right)$ & $\mathrm{g}_{\mathrm{x}}$ & $\mathrm{g}_{\mathrm{y}}$ & $\mathrm{g}_{\mathrm{z}}$ & Angle $\{\theta\}\left(^{\circ}\right)$ \\
\hline 12 & 15.17 & 0 & 0 & 14.42 & 98.59 \\
11 & 57.63 & 0 & 0 & 17.03 & 18.68 \\
10 & 28.37 & 0 & 0 & 16.77 & 35.97 \\
9 & $96.49^{*}$ & 0 & 0 & 17.42 & 39.17 \\
8 & $62.50^{*}$ & 0 & 0 & 17.61 & 60.46 \\
7 & 29.64 & 0 & 0 & 15.396 & 35.30 \\
6 & 19.89 & 0 & 0 & 14.36 & 9.55 \\
5 & 80.297 & 0 & 0 & 17.45 & 89.76 \\
4 & $336.92^{*}$ & 0 & 0 & 18.02 & 94.76 \\
3 & $505.80^{*}$ & 0 & 0 & 18.01 & 11.85 \\
2 & $308.48^{*}$ & 0 & 0 & 17.94 & 101.21 \\
\hline
\end{tabular}

*indicates barrier computed with second excited KDs due to collinearity with the first excited KDs.
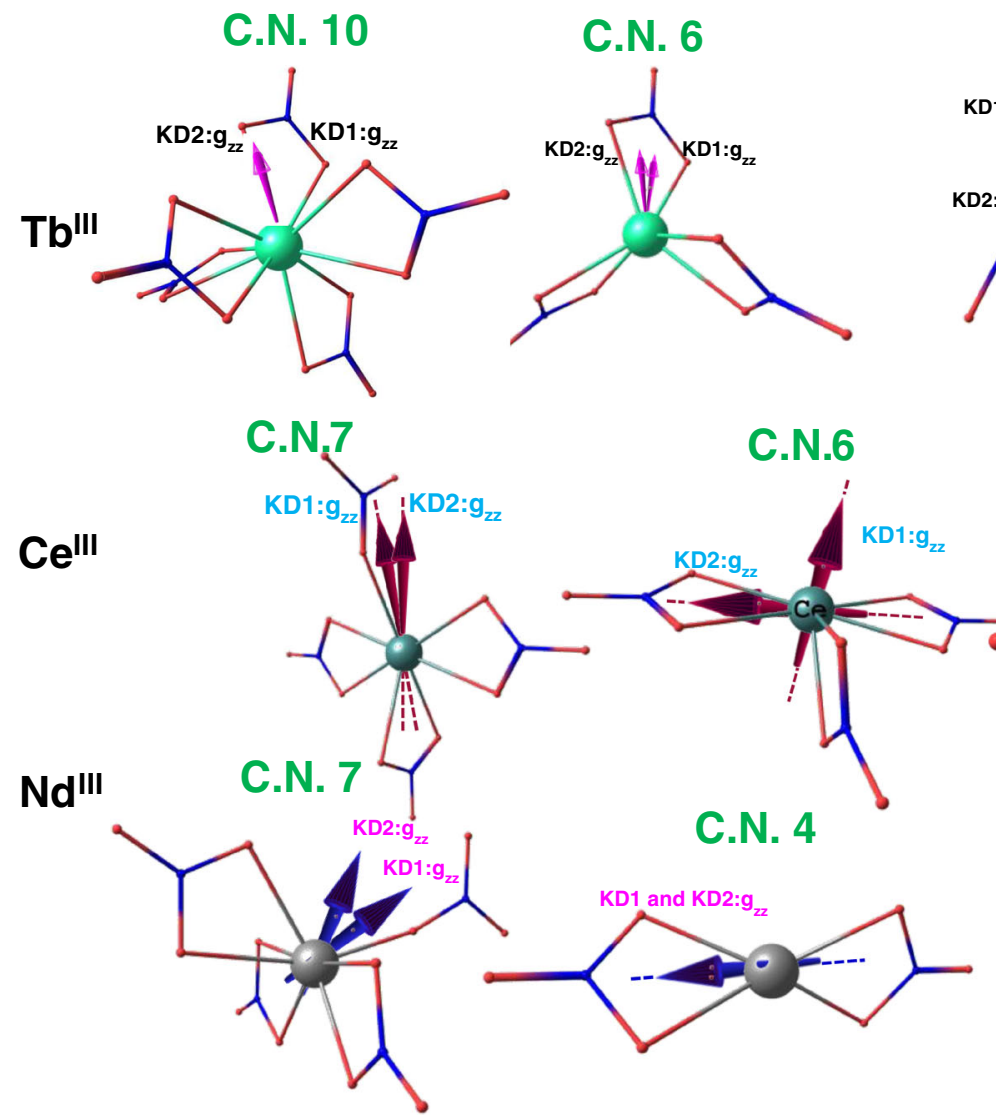

C.N. 3

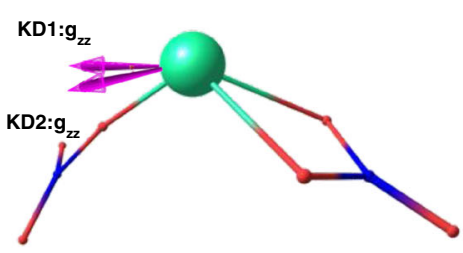

C.N. 2

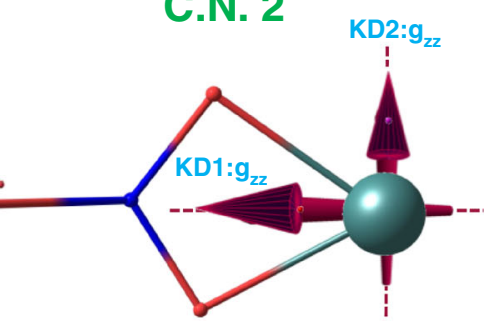

C.N. 2

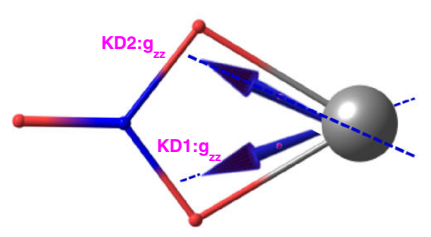

Figure 5. Model structures where $\mathrm{C}$. $\mathrm{N}$ is altered around $\mathrm{Tb}^{\mathrm{III}}, \mathrm{Ce}^{\mathrm{III}}$, and $\mathrm{Nd}^{\mathrm{III}}$ in $\mathbf{2}, \mathbf{3}$ and $\mathbf{4}$ along with the anisotropy directions of ground $v s$. first excited KDs/non-KDs.

have smallest $\theta$ angle (compared to other C. N. models). Models with C. N. 11 were found to possess rhombic anisotropy. As we decrease the C. N. around the $\mathrm{Nd}^{\mathrm{III}}$, a gradual increase in transverse nature of anisotropy is attained till C.N. 9. Further reduction of C. N. from 9 to 6 and 7 leads to an observation of rhombic nature of anisotropy. As we go further down from C.N. 5 to lower C. $\mathrm{N}$ number models, axial nature of the anisotropy is observed. The angle $\theta$ is found to be large for all tested models. 
Table 4. Calculated energy spectrum, g tensors and angles $(\theta)$ of the principal anisotropy axes of ground KDs of $\mathrm{Ce}^{\mathrm{III}}$ model complexes (from 11 to 2 C.N. change around $\mathrm{Ce}^{\mathrm{III}}$ ion) with respect to their respective first excited state KDs.

\begin{tabular}{lccccc}
$\begin{array}{l}\text { Coordination no. } \\
\text { around Ce }\end{array}$ & $\mathrm{U}_{\text {eff }}\left(\mathrm{cm}^{-1}\right)$ & $\mathrm{g}_{\mathrm{x}}$ & $\mathrm{g}_{\mathrm{y}}$ & $\mathrm{g}_{\mathrm{z}}$ & Angle $\{\theta\}\left(^{\circ}\right)$ \\
\hline 12 & 107.90 & 0.08 & 0.53 & 3.70 & 40.41 \\
11 & 48.91 & 0.57 & 1.31 & 3.56 & 78.27 \\
10 & 63.72 & 2.03 & 1.71 & 0.80 & 90.70 \\
$9^{\mathrm{a}}$ & 89.41 & 2.06 & 1.02 & 0.20 & 26.14 \\
7 & 431.76 & 0.54 & 0.59 & 3.72 & 14.78 \\
6 & 381.92 & 0.63 & 1.14 & 3.90 & 90.53 \\
5 & 299.14 & 2.46 & 2.24 & 0.83 & 20.19 \\
$4^{\mathrm{a}}$ & 821.28 & 0.05 & 0.06 & 4.16 & 89.30 \\
2 & 972.38 & 0.03 & 0.07 & 4.11 & 85.26 \\
\hline
\end{tabular}

a) note here that $\mathrm{C}$. $\mathrm{N}$ models 3 and 8 are missing as these models did not lead to minimum energy structures.

Table 5. Calculated energy spectrum, $g$ tensors and angles $(\theta)$ of the principal anisotropy axes of ground KDs of $\mathrm{Nd}^{\mathrm{III}}$ model complexes (from 11 to 2 C.N. change around $\mathrm{Nd}^{\mathrm{III}}$ ion) with respect to their respective first excited state KDs.

\begin{tabular}{lccccc}
\hline $\begin{array}{l}\text { Coordination no. } \\
\text { around Nd }\end{array}$ & $\mathrm{U}_{\text {eff }}\left(\mathrm{cm}^{-1}\right)$ & $\mathrm{g}_{\mathrm{x}}$ & $\mathrm{g}_{\mathrm{y}}$ & $\mathrm{g}_{\mathrm{z}}$ & Angle $\{\theta\}\left(^{\circ}\right)$ \\
\hline 12 & 16.20 & 0.58 & 1.73 & 3.66 & 85.30 \\
11 & 20.64 & 1.49 & 2.02 & 3.56 & 107.90 \\
10 & 48.18 & 2.83 & 2.69 & 1.81 & 47.14 \\
$9^{\mathrm{a}}$ & 37.24 & 2.30 & 2.49 & 1.44 & 47.94 \\
7 & 87.24 & 0.22 & 1.02 & 3.91 & 23.87 \\
$6^{\mathrm{a}}$ & 93.29 & 0.13 & 1.56 & 3.39 & 64.28 \\
$4^{\mathrm{a}}$ & 416.64 & 0.03 & 0.07 & 4.73 & $66.37^{*}$ \\
2 & 53.20 & 0.05 & 0.17 & 5.04 & 32.99 \\
\hline
\end{tabular}

a) note here that $\mathrm{C}$. $\mathrm{N}$ models 3,5 and 8 are missing as these models did not lead to minimum energy structures.

*indicates barrier computed with second excited KDs due to collinearity with the first excited KDs.

\subsection{Studies on complex 5 and 6}

The energy spectrum and g-tensors for two pseudodoublets and five singlets of the ground ${ }^{3} \mathrm{H}_{4}$ multiplet of $\mathrm{Pr}^{\mathrm{III}}$ ion in $\mathbf{5}$ are shown in table 1 with excited multiplet ${ }^{3} \mathrm{H}_{5}$ lying at $2270 \mathrm{~cm}^{-1}$. First three levels in $\mathbf{5}$ are singlet states and this precludes SMM behaviour for 5. It is noteworthy that, only fourth and fifth levels are pseudo doublets with magnetic moment and these are Ising in nature. The energy spectrum and g-tensors for three KDs of the ground ${ }^{6} \mathrm{H}_{5 / 2}$ multiplet of $\mathrm{Sm}^{\mathrm{III}}$ ion in 6 are shown in table 1 with excited multiplet ${ }^{6} \mathrm{H}_{7 / 2}$ lying at $1030 \mathrm{~cm}^{-1}$. Effective energy barrier required for reorientation of magnetization is found to be 90.03 $\mathrm{cm}^{-1}$ in complex 6 . Ground state anisotropy is rhombic in nature with first excited state possessing significant transverse anisotropy. Ground state KD lies at a large $\theta$ angle with respect to first excited KD in $\mathbf{6}$ resulting in possible relaxation via the first excited KDs.

\section{Conclusions}

We have performed fragment $a b$ initio calculations on a series of lanthanide complexes $\left[\operatorname{Ln}^{\mathrm{III}}=\mathrm{Dy}(\mathbf{1})\right.$, $\mathrm{Tb}(\mathbf{2}), \mathrm{Ce}(\mathbf{3}), \operatorname{Nd}(\mathbf{4}), \operatorname{Pr}(\mathbf{5})$ and $\operatorname{Sm}(\mathbf{6})]$ to shed light on the magnetic properties. Conclusions derived from our work are summarized below:

1) All the studied complexes except $\mathbf{5}$ are likely to exhibit SMM characteristics as significant barrier to reorientation of magnetisation is computed. However experimental observation of SMM behaviour may be hampered by QTM and intermolecular interactions. 
2) The computed $U_{\text {eff }}$ values for the five complexes are found to decrease in the order $(3>6>1>4>2$; $\mathrm{U}_{\text {eff }} \quad 107.9>90.03>27.93>16.2>15.17 \quad \mathrm{~cm}^{-1}$ ) suggesting largest barrier height for $\mathrm{Ce}^{\mathrm{III}}$ complex followed by $\mathrm{Sm}^{\mathrm{III}}$, Dy ${ }^{\mathrm{III}}, \mathrm{Nd}^{\mathrm{III}}$ and $\mathrm{Tb}^{\mathrm{III}}$. The $\mathrm{Dy}{ }^{\mathrm{III}}$ and $\mathrm{Sm}^{\mathrm{III}}$ are the best oblate and prolate ions respectively to exhibit SMM properties Thus it is not surprising that they possess large $\mathrm{U}_{\text {eff }}$ values. The $\mathrm{Ce}^{\mathrm{III}}$ ion possesses oblate electron density and is predicted to yield only small $\mathrm{U}_{\text {eff }}$ values. ${ }^{64}$ However here we predict that the $\mathrm{Ce}^{\mathrm{III}}$ structure possesses the largest $U_{\text {eff }}$ of the entire complex set tested. This is rather intriguing and unprecedented and requires an experimental verification.

3) Among the complexes studied, ground state anisotropy of complex $\mathbf{1}$ and $\mathbf{2}$ are Ising in nature while for $\mathbf{3}, \mathbf{4}$ and $\mathbf{6}$, a rhombic anisotropy is detected and complex $\mathbf{5}$ is not an SMM. The nature of anisotropy computed suggests that the tunnelling between the ground state KDs are likely to be small for complexes $\mathbf{1}$ and $\mathbf{2}$ while it is expected to be substantial for complexes $\mathbf{3}, \mathbf{4}$ and 6. This leads to larger QTM for 3, 4 and $\mathbf{6}$ while relatively less for $\mathbf{1}$ and $\mathbf{2}$ and this effect may overshadow the computed $\mathrm{U}_{\text {eff }}$ values discussed above.

4) Analysing the trend among the models with variation in C. N. reveals an inverse correlation between C. N. and $U_{\text {eff }}$ and $g_{z z}$ values. In all the tested cases low-coordinate models yield substantially higher barrier heights and this suggests that these are likely to be superior SMMs over regular high coordination number structures. Thus synthetic efforts targeted towards low-coordinate lanthanide complexes should be pursued to obtain larger $\mathrm{U}_{\text {eff }}$ values.

5) Among all the complexes tested three coordinate $\mathrm{Ce}^{\mathrm{III}}$ model is found to possess exceptionally large $\mathrm{U}_{\text {eff }}$ value of $972.38 \mathrm{~cm}^{-1}$ Although, it is challenging to synthesise low-coordinate $\mathrm{Ce}^{\mathrm{III}}$ complexes, extremely large barrier height computed might make them a suitable synthetic target.

\section{Supplementary Information}

Supplementary information contains coordinates of all the DFT optimised model complexes studied for complex 1, 3, 4 and $\mathbf{6}$ and computational details. For further details, visit www.ias.ac.in/chemsci.

\section{Acknowledgements}

GR would like to acknowledge financial support from the Government of India through the Department of Science and Technology (SR/S1/IC-41/2010; SR/ NM/NS-1119/2011) and Indian Institute of Technology, Bombay to access the high performance computing facility. TG would like to thank UGC New Delhi for JRF fellowship.

\section{References}

1. Leuenberger M N and Loss D 2001 Nature 410789

2. Saitoh E Miyajima H Yamaoka T and Tatara G 2004 Nature 432203

3. Yamanouchi M Chiba D Matsukura F and Ohno H 2004 Nature 428539

4. Evangelisti M Luis F de Jongh L J and Affronte M 2006 J. Mater. Chem. 162534

5. Bogani L and Wernsdorfer W 2008 NatMater 7179

6. Manoli M Collins A Parsons S Candini A Evangelisti M and Brechin E K 2008 J. Am. Chem. Soc. 13011129

7. Ganivet C R Ballesteros B de la Torre G Clemente-Juan J M Coronado E and Torres T 2013 Chem. Eur. J. 19 1457

8. Ishikawa N Sugita M and Wernsdorfer W 2005 Angew. Chem. Int. Ed. $\mathbf{4 4} 2931$

9. Christou G Gatteschi D Hendrickson D N and Sessoli R 2000 MRS Bull 2566

10. Jiang S D Wang B W Su G Wang Z M and Gao S 2010 Angew. Chem. Int. Ed. 497448

11. Jeletic M Lin P H Le Roy, J J Korobkov I Gorelsky S I and Murugesu M 2011 J. Am. Chem. Soc. 13319286

12. Jiang S D Wang B W Sun H L Wang Z M and Gao S 2011 J. Am. Chem. Soc. 1334730

13. Zhang P Guo Y N and Tang J K 2013 Coord. Chem. Rev. 2571728

14. Guo Z and Sadler P J 1999 Angew. Chem. Int. Ed. 38 1512

15. Bornhop D J Hubbard D S Houlne M P Adair C Kiefer G E Pence B C and Morgan D L 1999 Anal. Chem. 71 2607

16. Ishikawa N Sugita M Ishikawa T Koshihara S and Kaizu Y 2003 J. Am. Chem. Soc. 1258694

17. Ishikawa N Sugita M Ishikawa T Koshihara S and Kaizu Y 2004 J. Phys. Chem. B 10811265

18. Aromí G and Brechin E K 2006 Struct. Bond 1221

19. Gonidec M Luis F Vilchez A Esquena J Amabilino D B and Veciana J 2010 Angew. Chem. Int. Ed. 491623

20. Rinehart J D and Long J R 2009 J. Am. Chem. Soc. 131 12558

21. Guo Y N Xu G F Guo Y and Tang J K 2011 Dalton Trans. 409953

22. Car P E Perfetti M Mannini M Favre A Caneschi A and Sessoli R 2011 Chem. Commun. 473751

23. Rinehart J D Meihaus K R and Long J R $2010 \mathrm{~J}$. Am. Chem. Soc. 1327572

24. Guo Y N Xu G F Wernsdorfer W Ungur L Guo Y Tang J K Zhang, H J Chibotaru L F and Powell A K $2011 \mathrm{~J}$. Am. Chem. Soc. 13311948

25. Gatteschi D, Sessoli R J Villain 2006 In Molecular Nanomagnets (Oxford: Oxford University Press)

26. Sessoli R Gatteschi D Caneschi A and Novak M A 1993 Nature 365141 
27. Sessoli R and Powell A K 2009 Coord. Chem. Rev. 253 2328

28. Benelli C and Gatteschi D 2002 Chem. Rev. 1022369

29. Osa S Kido T Matsumoto N Re N Pochaba A and Mrozinski J 2004 J. Am. Chem. Soc. 126420

30. Zaleski C M Depperman E C Kampf J W Kirk M L and Pecoraro V L 2004 Angew. Chem. Int. Ed. 433912

31. Tang J K Hewitt I Madhu N T Chastanet G Wernsdorfer W Anson C E Benelli C Sessoli R and Powell A K 2006 Angew. Chem. Int. Ed. 451729

32. AlDamen M A Clemente-Juan J M Coronado E MartiGastaldo C and Gaita-Arino A 2008 J. Am. Chem. Soc. 1308874

33. Lin P H Burchell T J Clerac R and Murugesu M 2008 Angew. Chem. Int. Ed. 478848

34. AlDamen M A Cardona-Serra S Clemente-Juan J M Coronado E Gaita-Arino A Marti-Gastaldo C Luis F and Montero O 2009 Inorg. Chem. 483467

35. Gatteschi D and Sorace L 2001 J. Solid State Chem. 159 253

36. Zvezdin A K Matveev V M, Mukhin A A and Popov A I 1985 In Rare-earth ions in ordered magnetic crystals (Moscow: Nauka) p 296

37. Gudel H U Hauser U and Furrer A 1979 Inorg. Chem. 182730

38. Amoretti G Caciuffo R Carretta S Guidi T Magnani N and Santini P 2008 Inorg. Chim. Acta 3613771

39. Gatteschi D and Bencini A 1990 In EPR of Exchange Coupled Systems (Berlin: Springer-Verlag)

40. Barra A L Gatteschi D Sessoli R Abbati G L Cornia A Fabretti A C and Uytterhoeven M G 199 Angew. Chem. Int. Ed. 362329

41. Aquilante F De Vico L Ferre N Ghigo G Malmqvist $P$ A Neogrady P Pedersen T B Pitonak M Reiher M Roos B O Serrano-Andres L Urban M Veryazov V and Lindh R 2010 J. Comput. Chem. 31224

42. Bernot K Luzon J Bogani L Etienne M Sangregorio C Shanmugam M Caneschi A Sessoli R and Gatteschi D 2009 J. Am. Chem. Soc. 1315573

43. Cucinotta G Perfetti M Luzon J Etienne M Car P E Caneschi A Calvez G Bernot K and Sessoli R 2012 Angew. Chem. Int. Ed. 511606

44. Boulon M-E Cucinotta G Luzon J Degl'Innocenti C Perfetti M Bernot K Calvez G Caneschi A and Sessoli R 2013 Angew. Chem. Int. Ed. 125368

45. Gaussian 09, Revision D.01, Frisch M J, Trucks G W, Schlegel H B, Scuseria G E, Robb M A, Cheeseman J R, Scalmani G, Barone V, Mennucci B, Petersson G A, Nakatsuji H, Caricato M, Li X, Ratchian H P, Izmaylov A F, Bloino J, Zheng G, Sonnenberg J L, Hada M, Ehara M, Toyota K, Fukuda R, Hasegawa J, Ishida M, Nakajima T, Honda Y, Kitao O, Nakai H, Vreven T, Montgomery J A Jr., Peralta J E, Ogliaro F, Bearpark M, Heyd J J, Brothers E, Kudin K N, Staroverov V N, Kobayashi R, Normand J, Raghavachari K, Rendell A, Burant J C, Iyengar S S, Tomasi J, Cossi M, Rega N, Millam N J, Klene M, Knox J E, Cross J B, Bakken V, Adamo C, Jaramillo J, Gomperts R, Stratmann R E, Yazyev O, Austin A J, Cammi R, Pomelli C, Ochterski J W, Martin R L, Morokuma K, Zakrzewski V G, Voth G A, Salvador P, Dannenberg J J, Dapprich S, Daniels A D, Farkas Ö, Foresman J B, Ortiz J V, Cioslowski J, Fox D J Gaussian, Inc., Wallingford CT, 2009
46. Lee C T Yang W T and Parr R G 1988 Phys. Rev. B 37 785

47. Becke A D 1993 J. Chem. Phys. 985648

48. Stephens P J Devlin F J Chabalowski C F and Frisch M J 1994 J Phys Chem Us 911623

49. Cundari T R and Stevens W J 1993 J. Chem. Phys. 98 5555

50. Schafer A Huber C and Ahlrichs R 1994 J. Chem. Phys. 1005829

51. Chekhlov A N 2007 Russ. J. Inorg. Chem. 521741

52. del C Fernández-Fernández M Bastida R Macías A Pérez-Lourido P Platas-Iglesias C and Valencia L 2006 Inorg. Chem. 454484

53. Cui H Otsuka T Kobayashi A Takeda N Ishikawa M Misaki Y and Kobayashi H 2003 Inorg. Chem. 42 6114

54. Lu W-J Zhang L-P Chan H-S Chan T-L and Mak T C W 2004 Polyhedron 231089

55. Hamada T Manabe K Ishikawa S Nagayama S Shiro M and Kobayashi S 2003 J. Am. Chem. Soc. 125 2989

56. Wang Y-L Gu B, Ma Y Xing CWang Q-L Li L-C Cheng P and Liao D-Z 2014 CrystEngComm 162283

57. Ren M Pinkowicz D Yoon M Kim K Zheng L M Breedlove B K and Yamashita M 2013 Inorg. Chem. 52 8342

58. Chen G J Guo Y N Tian J LTang J Gu W Liu X Yan S P Cheng P and Liao D Z 2012 Chem. Eur. J. 182484

59. Binnemans K Malykhina L Mironov V S Haase W Driesen K Van Deun R Fluyt L Görller-Walrand C and Galyametdinov Y G 2001 Chem. Phys. Chem. 2 680

60. Bünzli J-C G and Piguet C 2002 Chem. Rev. 1021897

61. Murugesu M 2012 NatChem 4347

62. Blagg R J Ungur L Tuna F Speak J Comar P Collison D Wernsdorfer W McInnes E J L Chibotaru L F and Winpenny R E P 2013 Nat. Chem. 5673

63. Zhang P Zhang L Wang C Xue S Lin S-Y and Tang J 2014 J. Am. Chem. Soc. 1364484

64. Rinehart J D and Long J R 2011 Chem.Sci. 2 2078

65. (a) Herrmann W A, Anwander R, Munck F C, Scherer W, Dufaud V, Huber N W and Artus G R 1994 J Z Naturforsch. B 49 1789; (b) Bradley D C, Ghotra J S and Hart F A 1972 J. Chem. Soc. Chem. Commun. 349

66. Zhang P, Zhang L, Wang C, Xue S, Lin S-Y and Tang J 2014 J. Am. Chem. Soc. 1364484

67. Sundararajan M, Ganyushin D, Ye S and Neese F 2009 Dalton Trans 6021

68. (a) Duncan J A 2009 J. Am. Chem. Soc. 131 2416; (b) Aquilante F, De Vico L, Ferre N, Ghigo G, Malmqvist P A, Neogrady P, Pedersen T B, Pitonak M, Reiher M, Roos B O, Serrano-Andres L, Urban M, Veryazov V and Lindh R 2010 J. Comput. Chem. 31 224; (c) Veryazov V,Widmark P O, Serrano-Andres L, Lindh R and Roos B O 2004 Int. J. Quantum Chem. 100 626; (d) Karlstrom G, Lindh R, Malmqvist, P A, Roos B O, Ryde U, Veryazov V, Widmark P O, Cossi M, Schimmelpfennig B, Neogrady P and Seijo L 2003 Comp. Mater. Sci. 28 222

69. Malmqvist P A, Roos B O and Schimmelpfennig B 2002 Chem. Phys. Lett. 357230

70. Chibotaru L F and Ungur L 2012 J. Chem. Phys. 137 064112 